\title{
Gestion des catastrophes naturelles en sol québécois : rendre socialement et écologiquement responsables les processus de développement des territoires affligés
}

\author{
Diane Alalouf-Hall ${ }^{\mathrm{a}}$, Jean-Marc Fontan ${ }^{\mathrm{b}}$
}

RÉSUMÉ. Dans le contexte où les changements environnementaux induisent une augmentation des aléas météorologiques, les défis d'adaptation et de sécurité civile se multiplient. Cet article a été rédigé dans le cadre d'une thèse portant sur la réponse standardisée des acteurs lors de catastrophes d'origine naturelle. Nous nous intéressons à la gestion québécoise des catastrophes dites naturelles. D’abord, nous aborderons la question des limites juridiques de l'État québécois. Puis, nous nous pencherons sur la littérature portant sur le développement territorial. Cela nous permettra d'identifier la place occupée par la question de la prévention en matière de catastrophes naturelles. Enfin, nous présenterons un état de situation sur la question des zones inondables au Québec, ce qui nous permettra d'indiquer en quoi des mesures d'atténuation de la présence humaine habitée ont été pensées, mais peu prises en compte. Nous concluons en indiquant que le Québec ne peut se contenter d'innovations technologiques structurantes pour assurer un aménagement territorial qui soit socialement et écologiquement responsable face aux catastrophes naturelles.

\begin{abstract}
Where environmental changes increase weather hazards, the challenges of adaptation and emergency services increase. This article was written as part of a thesis on the standardized response of actors for natural disasters. We focus on Quebec's management of so-called natural disasters. First, we will address the specificity of the Quebec state, then, we will look at the literature on territorial development. This will allow us to identify the importance of prevention for natural disasters. Finally, we will present the situation on flood zones in Quebec, which will allow us to indicate how measures to mitigate inhabited human presence have been developed, but little were taken into account. We conclude by indicating that Quebec cannot be satisfied with structuring technological innovations to ensure territorial planning that is socially and ecologically responsible regarding natural disasters.
\end{abstract}

\section{Introduction}

Le réchauffement du système climatique est sans équivoque et le Québec n'est évidemment pas épargné. À l'horizon 2050, le collectif Ouranos (2015) prévoit une augmentation généralisée des températures moyennes sur tout le territoire québécois. Par ailleurs, les prévisions concernant les précipitations seront également en hausse au centre et au nord du Québec, avec un pic plus important durant les mois d'avril à octobre sur tout le territoire. Enfin, à l'horizon 2041-2070 et sur le plan de l'hydrologie, il faut s'attendre à une augmentation des débits hivernaux des rivières pour l'ensemble du Québec ainsi qu'à

\footnotetext{
a Doctorante en sociologie, Université du Québec à Montréal

b Professeur, département de sociologie, Université du Québec à Montréal
}

des crues printanières et des étiages estivaux et automnaux plus sévères (CEHQ, 2015).

Les signes du réchauffement climatique sont bien présents, et ce, depuis plusieurs décennies. La Base de données canadienne sur les catastrophes (BDC) ${ }^{1}$ recense plus de 1000 évènements survenus depuis 1900. Par catastrophe naturelle, il est entendu un évènement ayant tué des personnes (10 ou plus), touché ou affecté plus de 100 personnes, conduit la région affectée à demander de l'aide de la part du gouvernement canadien ou à l'international ou ayant entraîné des dommages ou des perturbations des activités à l'échelle d'une ou de plusieurs communautés. 
Dans cet article, nous nous intéresserons à l'enjeu de la gestion québécoise des catastrophes dites naturelles. Pour le Québec, les catastrophes naturelles les plus importantes sont principalement associées à des inondations, à des glissements de terrain, à des intempéries climatiques ou à des vagues de chaleur. De ces évènements, ceux qui retiennent particulièrement notre attention sont liés aux inondations.

Pourquoi cet intérêt pour les inondations? Pour au moins trois raisons. Premièrement, le phénomène est en hausse depuis les dernières décennies. Il s'agit donc de comprendre les raisons qui expliquent cette montée en importance. Deuxièmement, les catastrophes naturelles peuvent être classées en fonction de leur niveau de risque sur les humains. Si le territoire inondé n'avait pas été occupé, l'évènement se serait produit sans qu'il soit identifié comme ayant eu un impact sur des humains. Ce deuxième aspect pose donc une question simple qui demande réponse: pourquoi les autorités gouvernementales permettent-elles l'occupation résidentielle ou commerciale de territoires à risque d'être inondés? Troisièmement, les cas de catastrophes naturelles difficilement prévisibles mais possibles - par exemple, un tsunami ou un immense feu de forêt amènent des communautés locales à agir de façon préventive. C'est le cas au Japon, où des mesures ont été envisagées par la population locale afin, non pas de prévenir un tremblement de terre ou un tsunami, mais d'être mieux préparés au cas où un tel évènement advienne (Alalouf-Hall, 2019). Qu'en est-il pour le Québec? En quoi la littérature sur le développement territorial tient-elle compte de mesures préventives à adopter en réponse à l'enjeu des catastrophes naturelles?

Pour répondre à ces questions, nous nous pencherons, dans un premier temps, sur les limites juridiques de l'État provincial québécois. Deuxièmement, nous aborderons brièvement la littérature portant sur le développement territorial. Cela nous permettra d'identifier la place occupée ou non par la question de la prévention en matière de catastrophes naturelles. Troisièmement, nous présenterons un court état de situation sur la question des zones inondables au Québec, ce qui nous permettra d'indiquer en quoi des mesures d'atténuation de la présence humaine habitée ont été pensées, mais peu prises en compte, sous une forme cartographique, au moins depuis les années 1960.

\section{Les limites juridiques de l'État québécois}

La Constitution du Canada représente un cadre légal et politique : la loi des lois. Elle a été adoptée en 1867 par le Parlement de Londres. Cette constitution, régulée par l'ancienne métropole, a été rapatriée en 1982. Elle « définit les principales institutions politiques et juridiques d'un État. Pour le Canada, ce sont la Chambre des communes, le énat, la Cour suprême et les parlements des provinces, comme l'Assemblée nationale du Québec » (Par ici la démocratie, 2020, s. p.).

La Constitution canadienne a rendu possible l'existence de l'État canadien en tant que fédération d'un ensemble de territoires nommés "provinces». Ces territoires disposent d'importants champs de juridiction et, donc, de pouvoirs et de responsabilités spécifiques. Néanmoins, ces pouvoirs et responsabilités demeurent assujettis à une entité politique supérieure : celle du gouvernement du Canada. Mentionnons que la fédération canadienne met en scène trois paliers de gouvernement, lesquels sont imbriqués de façon verticale. Concrètement, le palier municipal relève de l'autorité des provinces, tandis que ces dernières sont assujetties à l'autorité fédérale en fonction d'un partage des pouvoirs et des responsabilités.

L'État canadien, par le biais du gouvernement du Canada, tout en se voulant un système politique décentralisé et déconcentré à l'échelle des provinces, n'en demeure pas moins régulé autour d'un noyau de compétences et de responsabilités qui concrétisent sa souveraineté. Quelles sont ces compétences et responsabilités? Elles relèvent d'éléments visant le bon fonctionnement de la fédération, dont : l'expansion de la fédération, la gestion des frontières (douanes), les relations commerciales à l'international, la monnaie et ses mécanismes de régulation, les voies maritimes, l'armée et tout ce qui touche aux questions relatives à la sécurité intérieure et extérieure ainsi qu'aux relations politiques avec d'autres pays ou grandes instances internationales.

Les autres champs de compétences et niveaux de responsabilités sont réservés aux provinces et aux territoires, lesquels gèrent ces espaces et leurs populations de concert avec les autorités municipales. Ces dernières ont des champs de compétences définis par l'autorité provinciale ou territoriale qui les enchâsse. 
Pour le Québec, la Loi sur les compétences municipales est la principale loi établissant les compétences et les pouvoirs des municipalités (Couture Ménard et Rioux Collin, 2019). En vertu de celle-ci, les municipalités locales peuvent agir dans cinq domaines : la culture; les loisirs; les activités communautaires et les parcs; le développement économique local; l'environnement et la sécurité.

Comme l'indiquent Couture Ménard et Rioux Collin (2019), afin d'assurer un cadre de vie adéquat et de qualité aux populations qui habitent leur territoire, les champs de compétences et les responsabilités des municipalités sont variés et importants. Cependant, les municipalités disposent de peu de moyens financiers ou de pouvoirs règlementaires pour bien assumer ces fonctions. De plus, elles doivent intervenir dans des champs de compétences qui ne cessent de se complexifier en fonction des pressions normatives visant leur développement, de la croissance des inégalités sociales, des mouvements migratoires, d'une évolution sociodémographique marquée par l'exode rural et par le vieillissement des populations, de la montée en importance des phénomènes identitaires, de l'augmentation du nombre de catastrophes naturelles et de la nécessaire adaptation à l'enjeu des changements climatiques.

Les réponses à apporter en matière d'urgence ou de prévention à la suite d'un évènement catastrophique relèvent du champ des compétences dévolues à la "sécurité publique intérieure 》 (Couture Ménard et Rioux Collin, 2019). Ce domaine de compétences, les municipalités le partagent avec les paliers gouvernementaux provincial et fédéral. Elles occupent cependant une position unique puisqu'elles représentent le premier niveau d'autorité publique en devoir de réponse aux besoins des populations affectées par une catastrophe naturelle.

Ainsi, le fait d'habiter un lieu sous juridiction d'une autorité municipale établit une relation directe de proximité entre les « habitants » d'une municipalité et cette unité publique. La relation avec les autres niveaux d'autorité est indirecte et transite par l'autorité municipale, laquelle, en cas d'urgence, doit faire appel aux autres paliers gouvernementaux pour mobiliser les ressources et les compétences requises afin de faire face à l'épreuve catastrophique ou d'en prévenir d'éventuelles.

Toute municipalité2, en raison de la Loi sur l'aménagement et l'urbanisme, se doit d'identifier les zones habitables ou développables à risque. Elle doit aussi définir les conditions et contraintes à respecter au moment d'une demande pour la délivrance d'un permis de construire. De façon complémentaire, toute municipalité est tenue d'entreprendre les travaux de prévention requis pour prévenir, quand faire se peut, une catastrophe naturelle. Concrètement, pour le Québec, il s'agit d'appliquer des normes de construction en fonction du risque que peut représenter un tremblement de terre ou encore d'empêcher toute localisation résidentielle, commerciale ou industrielle dans les zones sujettes à des glissements de terrain ou présentant des risques élevés en matière d'inondation.

Pour les municipalités, agir de façon préventive requiert des moyens financiers et des ressources. Ces moyens et ressources sont essentiels pour l'implantation de mesures propices à la résilience aux évènements catastrophiques (Davis et Alexander, 2016; Perras, 2019, Alalouf-Hall, 2019). Les actions à générer sont de quatre ordres : 1) actions de préservation des milieux naturels et mesures non technologiques (interdiction de vivre dans la zone protégée, forêts); 2) mesures structurelles ou technologiques du type systèmes de drainage des eaux pluviales, barrages, digues, bassins de rétention, rehaussement des sols, etc.; 3) actions informationnelles (cartographie, pédagogie en matière de sensibilisation et formation); et, enfin, 4) train des mesures temporaires en réponse à l'urgence d'une situation catastrophique.

À la différence des moyennes et grandes municipalités, les petites ne disposent pas des ressources requises. De plus, un désincitatif développemental a pour effet de ne pas empêcher toute localisation résidentielle, commerciale ou industrielle sur son territoire. «Dire non » signifierait priver une municipalité de revenus de taxation et ne faciliterait pas sa croissance territoriale ${ }^{3}$.

En regard de l'enjeu que représentent les catastrophes naturelles, depuis 1970, des accords d'aide financière en cas de catastrophe (AAFCC) ont été négociés entre le gouvernement fédéral et les provinces afin de légiférer en ce domaine. Les accords offrent « des secours aux sinistrés par l'entremise des gouvernements provinciaux et territoriaux » (Ministère de la Sécurité publique du Canada, 2007, p. 5). Ils ont essentiellement pour but «d'aider les provinces à couvrir les frais entraînés par une catastrophe si ceux-ci imposent à l'économie d'une province un fardeau trop important pour qu'on 
puisse raisonnablement s'attendre à ce qu'elle l'assume toute seule $[. .$. ] [et] visent à permettre de faire face aux catastrophes naturelles qui causent des dommages importants à la propriété ou l'interruption de la fourniture de biens et de services essentiels» (p. 5).

Les AAFCC concernent indirectement les municipalités. Spécifiquement, à la suite d'une catastrophe naturelle, il est indiqué qu'elles doivent transiger avec leur gouvernement provincial pour faire état de leurs besoins financiers. Il est clair que les accords sont imparfaits. Pour preuve, même si aucune mention n'est faite à cet effet, les municipalités, en cas de force majeure, peuvent en appeler directement au gouvernement fédéral afin de solliciter l'intervention des Forces armées canadiennes en cas d'urgence pour la sécurité publique de leur population.

Parler des modalités relationnelles prenant place entre des paliers de gouvernement nous situe dans le champ analytique de l'institutionnalisme étatique. Rappelons donc ce qu'est un État. Pour Jessop (1990), un État regroupe un «ensemble d'institutions et d'organisations [agissant collectivement], au nom d'une finalité socialement acceptée par les membres d'une société, qui consiste à définir et à appliquer, au nom de l'intérêt commun et d'une volonté générale, des décisions juridiquement contraignantes » (p. 341, trad. libre).

Dans la réalité du polymorphisme des formes de l'État, l'État canadien correspond à une forme spécifique de développement des États modernes, où s'est juxtaposée une capacité autonome réelle de construction étatique à partir d'une entité coloniale (p. ex., le Bas-Canada et le Haut-Canada ou encore la NouvelleÉcosse et Terre-Neuve) à une volonté plus ou moins franche de laisser s'opérer le processus de montée en souveraineté nationale desdites colonies par la métropole concernée (dans ce cas-ci, l'Angleterre). De la métropole, le Canada a hérité de solides institutions coloniales, sur lesquelles il a pu s'appuyer ou se distancer pour générer un processus et une dynamique de décolonisation qui furent réalisés relativement lentement et de façon pacifique et bienveillante. Par son histoire, par ses populations, par son mélange de cultures et par ses caractéristiques institutionnelles et organisationnelles, le Canada est une institution étatique spécifique, unique d'une certaine façon. Cet État-nation incarne une capacité spécifique et distincte de celle d'autres États de jouer son rôle et de remplir les fonctions qui lui sont dévolues.
En relation avec les évènements de plus en plus récurrents que représentent les catastrophes naturelles, comment évaluer la capacité générale d'intervention de l'État canadien?

Un premier élément de réponse nous est fourni par l'indice de classification des États. Selon l'Index des États fragiles, développé par Fund for Peace (2019), le Canada est considéré comme un État «very sustainable», donc comme un «État efficace » ou un «État fort». La notion d'État efficace fait référence à une évaluation de la capacité d'un acteur public d'être efficace et efficient. Les concepts d'État failli, d'État en crise, d'État effondré, d'État défaillant, d'État faible ou d'État fragile rendent compte de situations où est décelée « une incapacité [de l'État] à remplir les fonctions essentielles qui lui incombent, alors que la "capacité" englobe (a) la capacité organisationnelle, institutionnelle et financière de l'État à s'acquitter des fonctions essentielles associées au gouvernement d'une population et d'un territoire, et (b) l'aptitude d'un État à développer des relations constructives avec la société qui se renforcent mutuellement» (OCDE, 2010, p. 15).

Cet index permet de relativiser la notion d'État et d'aller au-delà de la classification proposée par Esping-Andersen (1990), laquelle distingue les États fondés sur un régime libéral, conservateur ou socialdémocrate. Dans les faits, les modalités historiques, contextuelles et conjoncturelles rendent les formes de l'État beaucoup plus diversifiées, complexes et fortement évolutives. Dès lors, être classé comme un État libéral ou social-démocrate permet certes de situer sur une échelle politique et idéologique une formation sociale par rapport à une autre, mais cela ne permet pas de comprendre la capacité réelle d'agir d'une formation sociale. L'index rappelle également que tous les États voient leur capacité ou incapacité évoluer, eu égard aux rôles et fonctions qui leur sont dévolus.

De la notion de «capacitation de l'État» en lien avec le territoire québécois et la « situation » engendrée par une catastrophe naturelle, nous proposons un modèle d'évaluation de cette capacitation qui tient compte des particularités de l'espace national canadien en matière de vulnérabilité et de résilience territoriales. En fonction de l'indice de capacité forte ou faible développée par Fund for Peace, le Canada est au nombre des pays les mieux classés, eu égard au respect des rôles et fonctions dévolus à un État. 


\begin{tabular}{|c|c|c|}
\hline Capacité effective d'intervention & Centre & Périphérie \\
\hline Forte & Canada & Singapore \\
\hline Faible & Russie & Haïti \\
\hline
\end{tabular}

Tableau 1 - Capacitation d'un État Source : Les auteurs

Il apparaît clairement que l'impact négatif d'une catastrophe naturelle sur un territoire sera d'autant plus grand que sera faible la capacité de résilience dudit territoire aux effets de la catastrophe. Dès lors, deux conclusions émergent de ce constat. D'une part, des territoires faiblement pourvus en capital socioterritorial seront fortement désavantagés par rapport à leur remise à niveau à la suite d'une catastrophe (en atteste l'État de la Louisiane à la suite de l'ouragan Katrina en 2005). Il s'ensuit alors une situation de défavorisation territoriale face aux catastrophes, ajoutant aux facteurs de défavorisation déjà existants.

Ainsi, pour l'espace canadien, la croissance des inégalités sociales engendre des inégalités territoriales (Klein et collab., 2010) qu'il faut prendre en considération, lesquelles sont observables à l'échelle des provinces, dans un premier temps, et des municipalités, dans un second temps. Certaines provinces sont mieux dotées en matière de développement territorial que d'autres.

\begin{tabular}{|c|c|c|}
\hline Capacité effective d'intervention & Centre & Périphérie \\
\hline Forte & Québec & X \\
\hline Faible & X & Terre-Neuve \\
\hline
\end{tabular}

Tableau 2 - Capacitation des provinces Source : Les auteurs

Cette réalité est aussi effective à l'échelle municipale. Les municipalités ont accès à des ressources et disposent de compétences inégales entre elles, ce qui joue dans leur capacité à bien répondre aux besoins d'une situation d'urgence générée par une catas- trophe naturelle. Alors interviennent d'autres considérations - d'ordre constitutionnel et politique dans la capacité effective de mobiliser adéquatement et rapidement les ressources à la disposition des paliers provinciaux ou fédéral de gouvernance.

\begin{tabular}{|c|c|c|}
\hline Capacité effective d'intervention & Centre & Périphérie \\
\hline Forte & Montréal & Chicoutimi \\
\hline Faible & Laval & Percé \\
\hline
\end{tabular}

Tableau 3 - Capacitation du Québec Source : Les auteurs

En prenant en considération l'importance de l'occupation humaine à l'échelle territoriale afin de générer du développement économique, certaines régions du Canada et du Québec sont avantagées par rapport à d'autres. Ainsi, une inondation, un feu de forêt, un glissement de terrain ou l'érosion d'une côte seront des évènements catastrophiques pour autant qu'ils mettent en danger des vies humaines. Des centaines de milliers d'hectares de forêt peuvent brûler sans que ce type d'évènement soit identifié comme catastrophique. Il en est ainsi avec l'érosion des côtes, où les seules situations suscitant une réaction préventive sont celles ayant directement un impact sur des populations humaines. Seront alors déployées des mesures d'enrochement ou de construction de murets afin de réduire l'effet érosif sur les zones à protéger.

Encore là, si des mesures sont appelées à être déployées, elles le seront si les territoires affectés seront jugés essentiels au "développement». Une analyse coût-avantage se trouve au cœur de la logique adaptative préconisée lorsqu'il s'agit de protéger un territoire, de l'adapter aux risques latents ou carrément de demander à la population concernée de le quitter.

Afin de faciliter les décisions et d'amorcer des actions de protection, d'adaptation, d'évacuation ou de relocalisation, le rôle des gouvernements provincial ou fédéral consiste à fournir le plus possible 
d'informations probantes et de qualité à la population et aux organisations concernées afin de les sensibiliser aux effets, par exemple, des changements climatiques sur leur milieu de vie. Ni le gouvernement fédéral ni ses vis-à-vis provinciaux ne peuvent intervenir directement sur l'enjeu de l'utilisation des sols. Il s'agit, constitutionnellement parlant, d'une compétence municipale. Ces ordres gouvernementaux produisent tout au plus des études, des rapports et des lois générales ou spécifiques afin d'aider les citoyens et les municipalités concernés à prendre des décisions éclairées.

Cette approche à responsabilité décentralisée, mais non imputable fait en sorte que, malgré la vulnérabilité attestée scientifiquement d'une localisation, le niveau de dangerosité d'une localisation n'est pas nécessairement pris en compte par les premiers concernés. Autant les dangers apparents d'inondation liés à la proximité d'un cours d'eau que l'histoire passée des inondations sont des facteurs qui devraient être pris en considération par les citoyens au moment d'une demande de permis de construire résidentiel, commercial ou industriel. Cette réalité est connue des autorités municipales, qui doivent en tenir compte dans l'étude de la demande de délivrance d'un permis. Dans les faits, ni le pressentiment de vulnérabilité ni l'évidence topographique ne sont réellement pris au sérieux.

Si la responsabilité de la décision de localisation en zone inondable incombe aux citoyens et aux municipalités, il n'en va pas de même de l'imputabilité de leurs actes. En cas de catastrophe naturelle, ces acteurs s'en référeront aux gouvernements supérieurs pour répondre favorablement à leur situation d'urgence. À moins de l'adoption et de l'implantation de règlements et de mesures particulières de la part des autorités supérieures ${ }^{4}$, une résilience s'installera à l'échelle locale, laquelle misera sur le fait de voir venir et de réparer, plutôt que de prévenir.

Si le Canada a les traits ou les attributs d'un État fort et, donc, s'il est capable de bien répondre à des urgences majeures à la suite d'une catastrophe naturelle, c'est principalement en comparaison aux États faibles, qui, eux, n'ont pas cette capacité et doivent largement compter sur l'aide internationale pour les soutenir lors de tels évènements. Dans les faits, nous sommes en présence de trois Canadas. Un Canada où :

- des zones centrales, fortement habitées et bien développées, bénéficient d'une attention et d'une protection maximales;

- des zones périphériques, peu habitées et moins développées, bénéficient d'une attention mitigée et minimaliste;

- des zones extra-périphériques, ne bénéficiant d'aucune attention ni couverture en cas d'urgence.

Les feux de forêt qui ont dernièrement affecté l'Arctique témoignent de la réalité d'un territoire canadien existant en bonne et due forme, mais non protégé. Sans impact direct sur des populations, ces feux laissent l'État fédéral indifférent. Pourtant, ces derniers contribuent à une augmentation des gaz à effet de serre et ont une incidence sur les dérèglements climatiques. Il en est ainsi de l'érosion côtière et des inondations affectant des zones très décentrées et présentant peu d'intérêt pour le développement économique.

\begin{tabular}{|c|c|c|c|c|}
\hline Capacité effective d'intervention & \multicolumn{3}{|c|}{ Centre } & Périphérie \\
\hline \multirow{3}{*}{$7^{\text {Forte }}$} & \multicolumn{3}{|c|}{ Canada } & Brésil \\
\hline & \multicolumn{2}{|c|}{ Zones habitées } & Zones extra- & \\
\hline & Centrale & Périphérique & & \\
\hline Faible & \multicolumn{3}{|c|}{ Russie } & Haïti \\
\hline
\end{tabular}

Tableau 4 - Capacitation territoriale inégale dans l'espace canadien Source : Les auteurs 
Il existe donc, au sein même de la fédération canadienne, des territoires protégés et des territoires orphelins de toute préoccupation préventive ou curative, eu égard aux catastrophes naturelles.

\section{Développement des territoires et rapport au territoire}

Au Québec, le constat est clair : devant la montée en importance des évènements catastrophiques, il apparaît nécessaire, pour les régions et les municipalités, de penser leur développement en des termes nouveaux. Nouveauté au sens où adaptation et résilience doivent impérativement nuancer le type de croissance socioéconomique souhaité ou attendu.

Mais voilà, les logiques du développement sont principalement pensées de façon descendante (du haut vers le bas), c'est-à-dire qu'elles sont fortement dépendantes de dynamiques et de processus qui échappent aux niveaux de pouvoir présents aux échelles locale et régionale. Nous l'avons vu en ce qui a trait aux catastrophes naturelles : les régions et les municipalités ont la responsabilité de répondre aux urgences, mais ne sont pas dotées des ressources requises pour agir et, qui plus est, elles ne sont pas réellement imputables aux actions à poser.

Pour assurer l'enrichissement des régions et des municipalités, le «développement des territoires » est le moyen privilégié par lequel l'humain moderne se dit en capacité de déployer toute sa grandeur. En fonction d'une telle configuration mentale, la nature devient une donnée passive. Son rôle et sa fonction sont d'être au service de l'humain afin de lui permettre de déployer pleinement sa grandeur. Nulle place, dans un tel esprit, pour une analyse écosystémique où l'humain serait amené à prendre en considération l'impact de son action sur l'environnement et, donc, à moduler cette dernière pour en réduire les effets négatifs. Pas étonnant, dans un tel contexte, que les théories du développement soient analphabètes sur l'enjeu écologique et myopes à l'égard des changements climatiques.

Les théories du développement font la promotion du progrès humain. Elles affichent un optimisme débordant, où le progrès n'a pas de date de péremption. Il est voué à un avenir éternel. Si cette façon de penser pouvait prévaloir par le passé, ce n'est plus le cas aujourd'hui. La date de péremption se rapproche de nous chaque jour et le développement à penser maintenant et pour le futur immédiat doit et devra être planifié en tenant compte de cette évidence éthique : notre soif de progrès demande à être étanchée et remplacée par une soif de «modestie » envers les progrès à faire advenir.

Notre « rapport au territoire » nous demande de moduler notre représentation de la notion de territoire. Elle ne peut plus se limiter à la seule définition du territoire comme entité anthropomorphique, uniquement au service de l'humain. Notre rapport au territoire demande à être élargie pour englober la «naturalité de l'espace», dans une perspective de « vivre avec », et non de « vivre contre le territoire ». Vivre avec le territoire sous-entend une posture écologique qui rende compte des droits, des obligations et des responsabilités éthiques et esthétiques qui nous lient profondément à la nature.

Est-ce possible? Ce possible, l'avons-nous déjà pris en compte?

Oui, tel est le sens profond du nomadisme et de la transhumance des sociétés premières de chasseurscueilleurs. Bouger et se déplacer afin de ne pas rendre les ponctions essentielles à la subsistance négatives aux écosystèmes occupés ou visités fait référence à un modèle de développement intégrant une éthique et une esthétique promouvant l'étendue et la durabilité des systèmes écologiques.

Aurait-il été possible de voir les sociétés humaines sortir du nomadisme en complexifiant leurs technologies sociales, tout en s'assurant de la préservation d'une relation saine et viable avec leur environnement écologique? Nous croyons que cela aurait été possible, et ce, à condition de bien réguler la montée en complexité sociétale par et dans du développement lent et juste sur les plans social et écologique. À condition aussi de réduire grandement nos désirs, besoins et aspirations conscients et inconscients à vouloir toujours être plus grands et plus puissants que nature. Il s'agissait donc d'opter pour une croissance non prédatrice, où le progrès se mesurerait par et dans des processus assurant l'étendue de la nature, et non une insatiable montée en grandeur d'élites humaines.

Concrètement, cette possibilité de développement lent est applicable aux zones dites inondables. Ces zones remettent en question le sacrosaint principe de sédentarité associé à la propriété privée et au 
capital encastré et valorisé par et dans ce type de propriété. Qu'est-ce à dire? Les zones à risque le sont parce que du capital économique est en danger de dévalorisation. Elles ne sont pas considérées comme à risque eu égard à la flore et à la faune. La définition du risque appliquée à ces zones est anthropocentrée. Le risque affecte du capital économique mis territorialement en valeur : des immeubles, des avoirs tangibles, des ressources, des infrastructures sociétales, etc. Si un immeuble mis en danger était mobile ou surélevé, il ne serait pas en danger de dévalorisation. Le phénomène d'inondation n'apparaitrait plus comme un problème. La zone ne serait plus dite à risque pour l'humain, mais elle le demeurerait toutefois pour la faune et la flore. Mais ça...!

\section{En territoires québécois, situation et enjeu des zones inondables}

Trop peu d'études ou d'ouvrages récents se sont penchés sur l'histoire des sinistres au Québec. Plusieurs études récentes ont constaté un écart entre la forte préoccupation des Québécois à l'égard des dérèglements climatiques et l'adoption réelle de comportements pour les prévenir et s'y adapter (Whitmore et Pineau, 2017; Bélanger et Gosselin, 2007). En 2018, le collectif Ouranos a conçu une base de données sur les sinistres survenus au Québec depuis le XVIIe siècle (MayerJouanjean et Bleau, 2018). La base est meublée de données provenant de sources écrites et iconographiques contenues dans les archives de la province.

Depuis le début des années 2000, l'ampleur des dégâts et les coûts engendrés par les facteurs de risque font en sorte que les préoccupations face à ces enjeux ont suscité diverses initiatives locales, tant sur le territoire québécois que sur l'île de Montréal (Therrien, Tanguay et Beauregard-Guérin, 2015). D'un point de vue scientifique, des centres universitaires ont spécialisé leurs études sur les dérèglements climatiques au regard de thèmes tels que la prévention ou la résilience. C'est le cas de Cité-ID LivingLab, de COPARI, du collectif Ouranos ou encore du Consortium ÉGIDE, lesquels fournissent des rapports précis et récents sur différents types de sinistres au Québec.

À l'échelle de la province, l'abondance des plans d'eau et des rivières rend le territoire québécois particulièrement exposé aux inondations. Régulièrement, de nombreuses collectivités font face à de tels évènements : le déluge du Saguenay en 19965, les grandes marées en décembre 2011, les inondations causées par le débordement de la rivière Richelieu et la tempête Irène en 2011, les débordements du ruisseau Pratt à Coaticook en 2014 et 2015, puis, tout dernièrement, les inondations printanières de grande ampleur de 2017 et de 2019 aux abords du fleuve Saint-Laurent et de ses affluents. Les crues printanières de 2017 ont touché 291 municipalités québécoises dans 15 régions administratives. Quelque 5300 résidences ont été affectées. Ces évènements ont entraîné l'évacuation d'environ 4000 personnes. Près de 400 routes ont été endommagées, alors que 22 municipalités et 1 agglomération ont déclaré l'état d'urgence (Ministère de la Sécurité publique du Québec, 2018a). À titre de comparaison, les crues de 2011 avaient atteint environ 40 municipalités. Concernant les coûts, ils sont également en hausse. Selon Larrivée, Sinclair-Desgagné, Da Silva, Desjarlais et Revéret (2015), les coûts moyens rattachés aux inondations ont été de l'ordre de $70 \mathrm{M} \$$ /an (en dollars de 2012) pour la période 1991-2013, mais les évènements extrêmes, tels que le Saguenay en 1996 et le Richelieu en 2011, ont élevé la facture à plus de 189 M\$ (en dollars de 2012).

Lorsqu'elles surviennent, les inondations rouvrent le débat sur la présence et la construction de bâtiments ou d'immeubles en zones inondables. Elles soulèvent de nouveau la question de la non-prise en compte des risques d'inondation dans les modalités d'aménagement du territoire. Elles remettent en évidence la vulnérabilité de plusieurs communautés et la nécessité de prendre des décisions pour réduire les risques.

Dans les territoires où elles surviennent, les inondations génèrent des pertes majeures et perturbent considérablement le fonctionnement normal des communautés affectées, induisant de plus en plus souvent des situations d'urgence. Conscient de sa responsabilité dans les domaines de la gestion des risques et de la protection des populations, le ministère de la Sécurité publique du Québec, en collaboration avec les acteurs locaux et régionaux, assure une surveillance continue de certains cours d'eau jugés à risque et travaille, avec des organismes d'urgence (p. ex., la Croix-Rouge), pour assurer la sécurité de la population affectée au moment d'une crise. Pour guider l'action des intervenants, de 
nombreux guides sont disponibles (Ministère de la Sécurité publique du Québec, 2018b; Croix-Rouge canadienne, 2018; Sous-comité de l'Organisation de la sécurité civile du Québec, 2015).

En matière de prévention, malgré les exemples nombreux démontrant l'inefficacité de telles mesures par rapport aux coûts engendrés, les réflexes de protection sont de construire des murs ou des digues. Ces mesures sont souvent privilégiées et encouragées par les municipalités. En 2014, la Ville de Gatineau a investi $2 \mathrm{M} \$$ pour améliorer de telles infrastructures dans un quartier le long de la rivière Gatineau (Beaudoin, 2018). Ces infrastructures incluent un système de barrages et de redressement de la bande riveraine. Basé sur les données des cinq plus importantes inondations, cet investissement était censé limiter les risques futurs d'inondation (St-Pierre, 2014). Aux vues de l'inondation du printemps 2017 et de ses effets, ce ne fut pas le cas.

À plus petite échelle, en avril 2019, la digue située près de Sainte-Marthe-sur-le-Lac a cédé, forçant l'évacuation de plus de 5000 personnes (RadioCanada, 2019). Depuis, les discussions ont repris concernant la reconstruction d'une digue plus efficace, répondant, selon les propos de Benoit Charrette, ministre de l'Environnement et de la Lutte contre les changements climatiques, aux «standards d'aujourd'hui » et « en prévention des crues du printemps $2020 »$ (Lorange, 2019).

Par ailleurs, les lois actuelles exigent qu'aucune structure ne soit construite dans la zone de crue de récurrence de 20 ans et qu'aucune ouverture (fenêtres ou portes) ne soit présente dans la zone de crue de 100 ans (MAMOT, 2017). Malgré cette prescription, toute construction bâtie avant l'entrée en vigueur des lois possède de facto des droits acquis qui permettent de contourner la loi. Dernièrement, le gouvernement du Québec a incité les résidents situés en zones inondables à se relocaliser. Un programme a été mis sur pied afin d'offrir une aide financière à la relocalisation. Les montants prévus sont souvent en dessous de la valeur foncière de certaines habitations en bord de rivière 6 . Les habitants se trouvent alors dans la situation délicate de devoir accepter une compensation financière trop basse par rapport à leur bien. Ils ont aussi le choix de refuser l'offre gouvernementale. En cas de refus, le Québec considérera que les résidents acceptent le risque et qu'ils recevront simplement un montant pour compenser les dommages de l'inondation printanière de 2019. Aucune assistance financière future ne sera octroyée en cas de sinistre après cette date.

Il est évident que le territoire québécois demande à être aménagé de façon à tenir compte des risques d'inondation. Plusieurs lois, politiques et règlements existent à cet effet. Toutefois, l'application du cadre légal en matière d'aménagement des plaines inondables n'est possible que si la cartographie est fiable, régulièrement mise à jour et formulée dans un langage clair et compréhensible pour faciliter les comparaisons et pour éviter toute confusion. Ces initiatives font écho à "l'impossible risque zéro » évoqué par Pigeon (2005), qui permet au moins de soulever des réflexions et de faire des remises en question sur le risque et sa gestion en intégrant la notion de coexistence selon le principe du vivre avec.

\section{Le difficile passage à un aménagement socialelement et écologiquement responsable}

L'histoire récente montre que les municipalités québécoises ont facilement obtenu des dérogations afin d'autoriser la localisation d'immeubles ou d'infrastructures dans des zones à risque ou sensibles. Chaque année, le ministère de l'Environnement et de la Lutte contre les changements climatiques autorise des dizaines de projets dans des plaines inondables et dans des milieux humides, et ce, malgré le fait bien connu du Ministère que ces plaines inondables agissent comme des zones tampons en période de crue. Agir contre la nature, la considérer comme un adversaire et non comme un "acteur» agissant et déterminant, n'est-ce pas semer les germes qui alimenteront la crise de demain? Sur ce point, il est clair que le Québec est appelé à connaître des inondations de plus en plus fréquentes et importantes dans leur ampleur.

Il est ainsi paradoxal de favoriser une amplification de la mise à risque de populations, tout en disant être en capacité d'éviter des crises à l'aide de demimesures (Therrien et collab., 2015). Or, il n'y a pas place à des demi-mesures. La gestion de ce type de crise nécessite non seulement une grande collaboration entre organisations publiques, privées et les 
acteurs de la société civile, mais aussi une compréhension élargie des actions post-crises à mettre en place afin de transformer les demi-mesures en mesures réellement préventives. Dans cette perspective, chaque épisode d'inondation représente une occasion d'apprendre à vivre avec la nature, et non contre elle. Agir ainsi permettra de réduire considérablement la portée et l'impact des risques tant sur nous que sur les écosystèmes naturels.

Dans cette veine, à la suite des inondations de 2017, la nécessité de doter la métropole montréalaise d'une vision claire face à l'enjeu des inondations a été perçue comme un élément fondamental pour assurer une bonne gestion des risques. La Communauté métropolitaine de Montréal $(\mathrm{CMM})^{7}$ a ainsi déployé une stratégie d'action. Elle a produit le Plan Archipel ${ }^{8}$, lequel est un cadre normatif inspiré des modèles français et anglais9. Il a pour fonction d'améliorer les connaissances relatives aux risques et aux vulnérabilités du grand Montréal. La CMM a aussi conclu une entente avec le gouvernement du Québec dans le cadre du Plan d'action en matière de sécurité civile relatif aux inondations. Cette entente délègue à la CMM des mandats de cartographie, de communication et de vulgarisation du risque ${ }^{10}$ en vue d'élaborer une nouvelle règlementation.

Bref, l'aménagement sécuritaire des territoires demande à être pensé à différentes échelles, à la fois temporelles et spatiales, afin d'éviter la maladaptation aux risques. En ce sens, la cartographie des risques représente un processus de veille incontournable permettant de recenser les risques, de les différencier et de suivre l'évolution des niveaux de risque. En tant que mesure de veille, cette montée en connaissance est indispensable au travail d'analyse requis pour bien gérer l'aménagement et le développement des territoires.

Toutefois, cédant à la pensée magique selon laquelle la nature est domesticable et contrôlable par l'humain, et que ce dernier a la capacité de mobiliser les savoirs et les technologies requis pour assurer la sécurité de populations, certaines municipalités, aidées par le gouvernement fédéral, investissent beaucoup plus dans la prévention centrée sur des technologies dures, à l'image des digues ou de l'enrochement de berges, que sur des mesures souples et compréhensives des situations. Ces mesures miseraient plus sur l'évitement, le contournement, le respect ou la mise en place de zones d'atténuation ou de zones tampons.

Les technologies dures, proposées par les firmes d'ingénierie, sont séduisantes pour les personnes élues. Elles laissent entendre qu'il est possible d'assurer un aménagement sécuritaire pour le territoire, indépendamment de l'ampleur des risques. Cette fausse certitude laisse entendre que le génie technologique représente LA solution adéquate aux débordements de la nature, et ce, peu importe l'ampleur de ces derniers. S'il est vrai qu'il y a une efficacité certaine à ces technologies, elle n'est pas absolue et demande à être complétée par des mesures souples de prévention en amont. De telles mesures dynamiques sont implantées en amont et se déploient pendant la catastrophe et en suivi à celle-ci.

À titre indicatif, ériger une digue sans développer un cadre social de prévention alimente un sentiment erroné de sécurité. L'exemple de Kamaishi au Japon est révélateur en ce sens. Malgré la présence d'un mur brise-lames qui a coûté des milliards de dollars, les écoliers et écolières ont suivi des cours de prévention aux catastrophes (en amont de tout évènement), les rendant beaucoup mieux avisés sur les choses à faire que leurs ainnés au moment où le tsunami a frappé la ville (Alalouf-Hall, 2019). Le Québec, comme c'est le cas au Japon, ne peut se contenter d'innovations technologiques structurantes pour assurer un aménagement territorial qui soit socialement et écologiquement responsable face aux catastrophes naturelles.

\section{Conclusion prospectiviste}

En guise de conclusion, nous avançons trois pistes d'action pour le Québec.

Premièrement, une révision du cadre normatif de la gestion des risques et des inondations s'impose. Le cadre normatif à implanter devrait s'appuyer sur des mécanismes renouvelés de gouvernance qui permettraient une application uniforme et cohérente de la part des différentes instances locales, régionales et gouvernementales. Sur ce point, une révision des pouvoirs constitutionnels est requise afin de transférer plus de capacités d'agir et de reconnaittre plus d'imputabilité aux municipalités. 
Deuxièmement, la gestion des risques, dans le cas précis des inondations, ne peut plus uniquement reposer sur une vision mécanique des risques d'inondation, mais aussi sur une compréhension des vulnérabilités territoriales. Il s'agit donc de prendre pleinement en considération les dimensions sociales et écologiques au moment de développer et d'aménagement des territoires. Une composante sociale en accord avec les inégalités en capacités qui différencient les différents territoires du Québec. Une composante écologique en fonction d'une prise en compte des particularités des écosystèmes en présence. De plus, il importe de renouer avec les savoirs locaux - présents et passés
-, lesquels sont très importants pour faire face aux pressions au développement venant de l'extérieur et pour les moduler.

Agir selon ces deux principes en appelle un troisième : changer l'esprit du temps. La résilience face aux risques d'inondation implique de laisser la place à l'eau et à la nature, comme l'indique l'expérience du Plan Archipel, et non de considérer l'eau comme un ennemi à contrôler par le déploiement de technologies de plus en plus sophistiquées. Il s'agit donc de travailler avec la nature, plutôt que de vouloir à tout prix la contrôler et la soumettre aux impératifs du développement.

\section{NOTES}

1 Pour la BDC, voir : www.securitepublique.gc.ca/cnt/rsrcs/cndn-dsstr-dtbs/index-fr.aspx. Pour une base de données publiée par le gouvernement du Québec, voir : www.donneesquebec.ca/fr/2016/11/21/evenements-catastrophes-naturelles-a-lere-donneesouvertes.

2 Pour plus de précisions, voir Ministère de la Sécurité publique du Québec (2014).

3 Sur la non-prise en compte des cartographies des zones inondables par les municipalités, voir Verville (2018).

4 À l'image de certaines actions et de certains programmes ponctuels mis en place pour aider des régions et municipalités aux prises avec des problèmes d'érosion des berges. Sur cette question, voir Robillard (2019).

5 Cet évènement a causé la mort de 10 personnes. C’est également la première catastrophe d'origine naturelle qui a généré plus de $1 \mathrm{G} \$$ de dommages au Canada (Ouranos, 2018).

6 En 2015, le prix d’une propriété au bord du fleuve dans la région de la Capitale-Nationale était de 400000 à $500000 \$$ (jusqu’à $1 \mathrm{M}$ \$ et plus pour des propriétés de luxe) (Rougerie, 2015).

7 La CMM est un organisme de planification, de coordination et de financement à caractère métropolitain. Elle représente 82 municipalités du grand Montréal. Voir : www.cmm.qc.ca.

8 https://cmm.qc.ca/planification/plan-archipel

9 Paris et Londres sont fortement exposées aux risques d'inondation.

10 Dont la production d'une trousse d'information pour les municipalités et la population.

\section{RÉFÉRENCES}

Alalouf-Hall, D. (2019). « Le miracle de Kamaishi » : les enseignements du tsunami de 2011 au Japon. Alternatives humanitaires, 10 , 148-161. Repéré à http:/ / alternatives-humanitaires.org/fr/2019/03/25/le-miracle-de-kamaishi-les-enseignements-du-tsunamide-2011-au-japon

Beaudoin, M. (2018). Analyse des solutions possibles aux inondations dans le centre urbain d'Ottawa et de Gatineau (Mémoire de maitrise). Université de Sherbrooke, Sherbrooke, Québec. Repéré à https://savoirs.usherbrooke.ca/bitstream/handle/11143/11893/ Beaudoin_Michelle_MEnv_2018.pd??sequence=1\&isAllowed=y

Bélanger, D. et Gosselin, P. (2007). Changements climatiques au Québec méridional : perceptions de la population générale et suggestions d'adaptations futures. Montréal, QC : Institut national de santé publique du Québec. Repéré à www.inspq.qc.ca/sites/default/files/ publications/618-changclimatmeridionalpercep.pdf

Centre d'expertise hydrique du Québec (CEHQ). (2015). Atlas hydroclimatique du Québec méridional : impact des changements climatiques sur les régimes de crue, d'étiage et d'bydraulicité à l'horizon 2050. Montréal, QC : CEHQ. Repéré à www.cehq.gouv.qc.ca/ hydrometrie/atlas/atlas_hydroclimatique.pdf

Couture Ménard, M.-E et Rioux Collin, J. (2019). Les compétences et les pouvoirs des municipalités pour créer des environnements favorables à la saine alimentation et au mode de vie physiquement actif. Québec, QC : Institut national de santé publique du Québec. 
Croix-Rouge canadienne. (2018). Guide sur le rétablissement à la suite d'une inondation. Repéré à www.croixrouge.ca/crc/documentsfr/ Guideretablissement-a-la-suit-inondation.pdf

Davis, I. et Alexander, D. (2016). Recovery from disaster. New York, NY: Routledge.

Esping-Andersen, G. (1990). The three worlds of welfare capitalism. Princeton, NJ: University Press.

Fund for Peace. (2019). Fragile States Index 2019. Washington, DC: Fund for Peace. Repéré à https:/ / fundforpeace.org/2019/04/10/fragile-states-index-2019

Jessop, B. (1990). State theory: Putting the capitalist state in place. Cambridge, R.-U.: Polity Press.

Klein, J.-L., Fontan, J.-M., Saucier, C., Tremblay, D.-G., Tremblay, P.-A. et Simard, M. (2010). Les conditions de réussite des initiatives locales de lutte contre la paurreté et l'exclusion qui mobilisent des ressources de l'économie sociale (cahier no ET1002). Montréal, QC : Centre de recherche sur les innovations sociales. Récupéré à https:// crises.uqam.ca/wp-content/uploads/2018/10/ET1002.pdf

Larrivée, C., Sinclair-Desgagné, N., Da Silva, L., Desjarlais, C. et Revéret, J.-P. (2015). Évaluation des impacts des changements climatiques et de leurs coûts pour le Québec et l'État québécois (Rapport d'étude préparé pour le Bureau de projet des changements climatiques). Montréal, QC : Ouranos. Repéré à www.environnement.gouv.qc.ca/changementsclimatiques/evatuation-impacts-cc-couts-qc-etat.pdf

Lorange, S.-O. (2019, 10 mai). Une nouvelle digue pour Sainte-Marthe-sur-le-Lac. La Presse. Repéré à www.lapresse.ca/actualites/ politique/201905/10/01-5225515-une-nouvelle-digue-pour-sainte-marthe-sur-le-lac.php

Mayer-Jouanjean, I. et Bleau, N. (2018). Historique des sinistres d'inondations et d'étiages et des conditions météorologiques associées (Rapport présenté à Environnement et Changements climatiques Canada et au gouvernement du Québec). Montréal, QC : Ouranos. Repéré à www.ouranos.ca/publication-scientifique/RapportMayerJouanjean2018.pdf

Ministère de la Sécurité publique du Canada. (2007). Lignes directrices sur les Accords d'aide financière en cas de catastrophe. Ottawa, ON : Gouvernement du Canada.

Ministère de la Sécurité publique du Québec. (2014). Rôle et responsabilités des principaux intervenants en matière de prévention. Québec, QC : Gouvernement du Québec. Repéré à www.securitepublique.gouv.qc.ca/securite-civile/surveillance-du-territoire/ responsabilites-securite-civile.html\#c22193

Ministère de la Sécurité publique du Québec. (2018a). Préparer la réponse aux sinistres : guide à l'intention du milieu municipal pour l'établissement d'une préparation générale aux sinistres. Québec, QC : Gouvernement du Québec. Repéré à www.securitepublique.gouv.qc.ca/ fileadmin/Documents/securite_civile/soutien_partenaires/guide_reponse_sinistres_2018-07-19.pdf

Ministère de la Sécurité publique du Québec. (2018b). Plan d'action en matière de sécurité civile relatif aux inondations. Québec, QC : Gouvernement du Québec. Repéré à www.securitepublique.gouv.qc.ca/fileadmin/Documents/securite_civile/ inondation/Plan_action_inondations.pdf

Ministère des Affaires municipales et de l'Occupation du territoire du Québec (MAMOT). (2017). Inondations printanières 2017 : aménagement du territoire. Québec, QC : Gouvernement du Québec.

Organisation de coopération et de développement économiques (OCDE). (2010). La légitimité de l'État dans les situations de fragilité: analyser la complexité. Paris, France : OCDE.

Ouranos. (2015). Vers l'adaptation: synthèse des connaissances sur les changements climatiques au Québec. Montréal, QC : Ouranos. Repéré à www.ouranos.ca/publication-scientifique/SyntheseRapportfinal.pdf

Par ici la démocratie. (2020). Qu'est-ce qu'une constitution? Repéré à www.paricilademocratie.com/approfondir/territoire-etconstitutions/44-qu-est-ce-qu-une-constitution-

Perras, C. (2019). La reconstruction et le réaménagement dans la plaine inondable après les inondations du printemps 2017 à deux- montagnes : quelles leçons pour l'aménagement résilient du territoire? Université de Montréal.

Pigeon, P. (2005). Géographie critique des risques. Paris, France : Economica-Anthropos.

Radio-Canada (avec les informations de Jacaudrey Charbonneau) (2019, 5 mai). La digue de Sainte-Marthe-sur-le-Lac bientôt réparée. ICI Grand Montréal. Repéré à https://ici.radio-canada.ca/nouvelle/1167995/sainte-marthe-lac-colmatage-digue-naturelle

Robillard, J.-P. (2019, 16 janvier). Québec indemnisera des victimes des changements climatiques au Bas-Saint-Laurent. ICI Bas-SaintLaurent. Repéré à https:/ /ici.radio-canada.ca/nouvelle/1147287/indemnisation-victime-changement-climatique-bas-saint-laurentsainte-flavie-berge-maree-erosion 
Rougerie, M. (2015, 6 juin). Quelques secrets au bord du Saint-Laurent. La Presse+. Repéré à http://plus.lapresse.ca/screens/c2a191f2-389d-4f7d-b12e-1080bc1a9cb8_7C__ $0 . h t m l$

Sous-comité de l'Organisation de la sécurité civile du Québec (2015). L'intervention d'ungence hors du réseau routier : cadre de réference. Québec, QC : Gouvernement du Québec. Repéré à www.securitepublique.gouv.qc.ca/securite-civile/publications-et-statistiques/ lintervention-durgence-hors-du-reseau-routier-cadre-de-reference/en-ligne.html

St-Pierre, G. (2014, 3 avril). Gatineau entame des travaux afin de réduire les inondations. Le Droit. Repéré à https://www.ledroit.com/actualites/gatineau/gatineau-entame-des-travaux-pour-reduire-les-inondations-75a7610f8fb2dd21ebb5cb50de0b2011

Therrien, M.-C., Tanguay G. A. et Beauregard-Guérin, I. (2015). Fundamental determinants of urban resilience: A search for indicators applied to public health crisis. Resilience, 3(1), 18-39. https://doi.org/10.1080/21693293.2014.988915

Verville, A. (2018, 16 août). Cartographie des zones inondables : 35 ans plus tard. Le Soleil. Repéré à www.lesoleil.com/ opinions/point-de-vue/ cartographie-des-zones-inondables-35-ans-plus-tard-5956730a6163202c55bd791eed5412e3

Whitmore, J. et Pineau, P.-O. (2017). État de l'énergie au Québec 2018. Montréal, QC : Chaire de gestion du secteur de l'énergie, HEC Montréal. Repéré à https://energie.hec.ca/wp-content/uploads/2017/12/EEQ2018_WEB-FINAL.pdf 